\title{
A New Choroidal Layer Segmentation Method Based on Graph Theory and Directional Curvelet Transform in OCT Images
}

\author{
Mahdad Esmaeili \\ Tabriz University of Medical Sciences \\ Reza Alizadeh Eghtedar \\ Tabriz University of Medical Sciences \\ Alireza Peyman \\ Isfahan University of Medical Sciences \\ Mohammadreza Akhlaghi \\ Isfahan University of Medical Sciences
}

Seyed Hossein Rasta ( $\nabla$ s.h.rasta@abdn.ac.uk)

Tabriz University of Medical Sciences

Research article

Keywords: Choroidal segmentation, Optical coherence tomography, Image processing, Graph theory, Curvelet transform

Posted Date: December 4th, 2019

DOI: https://doi.org/10.21203/rs.2.18088/v1

License: (c) (1) This work is licensed under a Creative Commons Attribution 4.0 International License. Read Full License 


\section{Abstract}

Background: Automatic segmentation of the choroid on Optical Coherence Tomography (OCT) images helps ophthalmologists in diagnosing eye pathologies. In nature, it is not as exhausting as manual segmentation and does not depend on human errors. In this study, sixty EDI-OCT (Enhanced Depth Imaging Optical Coherence Tomography) images of both normal and abnormal eyes gathered from Isfahan Feiz Medical Center were used. The data were manually segmented by a retinal ophthalmologist to draw comparison with the proposed automatic segmentation technique.

Methods: In this study, curvelet transform based KSVD dictionary learning and Lucy-Richardson algorithm was used to remove speckle noise from OCT images. The Outer / Inner Choroidal Boundaries (O/ICB) were determined utilizing graph theory. The area between ICB and OCB was considered as choroidal region.

Results: The method was evaluated on the EDI-OCT images and the average Dice Similarity Coefficient (DSC) was calculated to be $92.14 \% \pm 3.30 \%$ between automatic and manual segmented regions. Moreover, by applying the latest presented open-source algorithm by Mazzaferri et al on our dataset the mean DSC was calculated to be $55.75 \% \pm 14.54 \%$.

Conclusions: A significant similarity was observed between automatic and manual segmentations, in both normal and abnormal eyes. Automatic segmentation of the choroidal layer could be also utilized in large-scale quantitative studies of the choroid.

\section{Background}

The choroid layer (the distance between the Outer Choroidal Boundary (OCB) and the Inner Choroidal Boundary (ICB) is a vascular structure in the posterior part of the eye between sclera and retina and possesses some key roles in eyes including oxygen supply and feeding of the retina (Figure 1). Several parameters such as age and some of the eye diseases affect the thickness of choroid (1-4).

Optical Coherence Tomography (OCT) is a novel non-invasive imaging technique, which is applied to the imaging of the human retina (5). the Enhanced Depth Imaging Optical Coherence Tomography (EDI-OCT) introduced by Spaide et al. (2015) obtains high-resolution cross-sectional images of the choroid and has been used in recent works on choroidal segmentation (6).

The main objective of automatic choroidal segmentation is to help ophthalmologists to monitor and diagnose eye diseases. This fact has made many researchers, all around the world, to focus on the subject matter. Study of current methods shows that there are a few reliable commercially available software to automatically segment the choroidal layer. So most of the ophthalmologists segment this layer using manual or semi-automatic techniques. The inhomogeneous intensity of choroidal layer, low contrast of OCT images, and the presence of speckle noise has made the automatic choroidal segmentation to be a challenging task $(7,8)$. In order to reduce speckle noise from OCT images, many 
traditional methods such as adaptive median and Wiener filtering $(9,10)$, median and Lee filtering (1114) are suggested but these methods are often obscure in details and affect edges in an image. In this paper we have used a new 2-Dimensional (2D) curvelet-based K-SVD algorithm (15) to speckle noise reduction. Despite the fact that this method enhances intraretinal layers, with noise suppression and optimally despeckling OCT image, the Texture Preservation (TP) parameter, which is a measure of retaining texture in a Region of Interest (ROI), seems not to be satisfactory (TP would be close to 0 for severely flattened image and remains close to 1 at its best). Therefore, to correct these weaknesses and deblurring of the resulted images, we used an efficient Lucy-Richardson deconvolution algorithm (16) to enhance intra-retinal boundaries and deblur the resulted images. Also, we introduced a new method based on graph theory and curvelet transform, for automatic segmentation of the choroid in OCT images. The experimental results showed that automated extracted regions significantly matched with the manual segmentations, helped the ophthalmologists in recognizing eye abnormalities (17). The comparison of results has shown great superiority of this presented method over other proposed methods. Table 1 shows the reported Dice Coefficient in other studies and introduces them briefly.

The challenging issues associated with automatic choroidal segmentation in OCT images include the depth of choroidal tissue, the vascular and heterogeneous structure of this layer that makes recognition of the layer difficult task (7). In this study, we try to cope with the mentioned challenges and present a new and fully-automated technique for the segmentation of choroid in EDI-OCT images. To prevent inaccurate detection of choroidal boundaries, at first, the OCT images are preprocessed and the reflective layers above the Bruch's membrane are removed, then the curvelet transform is used and curvelet coefficients are modified to enhance and fill the black shadowing effects. The graph-based segmentation of ICB and OCB layers may be degraded because of the presence of these possible gaps due to large vessels.

\section{Methods}

Un-preprocessed OCT images, like ultrasound images, do not have smooth appearances because of the presence of speckle noise that pollutes image features. By denoising OCT images, images quality can be improved leading to an accurate analysis of intra-retinal layers including choroidal layer. In this study, a 2D curvelet-based K-SVD algorithm (12) was used to speckle noise reduction of OCT images. Although this method has an acceptable performance, the TP parameter was not satisfactory and the resulted images suffered from blurring effects, thus, an effective Lucy-Richardson's deconvolution algorithm (13) was used to enhance and deblur the resulted images.

Lucy-Richardson's deconvolution algorithm was originated from the theorem of Bayes by Lucy and Richardson in the 1970 s $(18,19)$. Among the various deconvolution algorithms being investigated in different studies, Lucy-Richardson has been reported to be the algorithm with the most prosperous results $(16,20,21)$. This algorithm is commonly used due to its ability in recovering blurred and noisy images, which have been blurred by a known Point Spread Function (PSF) (16). The main formula of the algorithm is as follows(16): 
See supplemental file for formula 1

Where:

gnx,y is the undistorted image estimation in the $\mathrm{n}^{\text {th }}$ iteration. The process of enhancement starts with $g 0 x, y=k(x, y)$ and improves gnx,y based on the original output image $k(x, y)$, and $u(x, y)$ is the PSF of the imaging system, Figure 2 shows the block diagram of the proposed method for OCT image despeckling.

In this study, an automatic algorithm, based on Dijkstra's shortest path graph-search algorithm, that was fully described in following references $(22,23)$, is presented to segment the inner choroidal boundary (ICB). The Dijkstra's algorithm uses paths with minimum weigh, and to help the graph to follow the ICB, there is a need to add an additional column of nodes with minimal weights (wmin) to both sides of the image, which can be removed after segmentation. The following formula shows weight of the edges which connect adjacent nodes $\mathrm{i}$ and $\mathrm{j}(7)$;

See supplemental file for formula 2

Where:

Wij is the weight assigned to the edge connecting adjacent nodes $i$ and $j$, wmin $=1 \times 10-5$ is the minimum weight in the graph, and gi, gj are the gradient information at node $i$ and $j$. An example of the automatic segmentation of ICB is shown in Figure 3.

As mentioned earlier, the automatic detection of OCB is a less challenging task, due to the depth of choroidal tissue which makes this layer to be non-uniform (7). In this study, the histogram of each OCT image was defined at a particular threshold based on the mean brightness of each image as shown in Figure 4-A. The pixels with maximum value in each column of the resulted images were found and a third-degree curve was assigned to these pixels that was considered as OCB. Figure 4-B shows an example of the automatic segmentation of OCB.

\section{Results}

The proposed preprocessing algorithm was examined on 17 publically available 2D-OCT images (24) of eyes with and without non-neovascular Age-related Macular Degeneration (AMD) and 60 EDI-OCT images of both normal and abnormal eyes obtained from Isfahan Feiz Medical Center were utilized to evaluate the proposed automatic choroid segmentation method. The dictionary size was $16 \times 256$ for the coefficient matrix in scale 7 and was $16 \times 128$ used for denoising the curvelet coefficient matrix in scale 6 . A qualitative performance of our new proposed method on two different OCT images has been illustrated in Figure 5.

At first, the Edge Preservation (EP), Texture Preservation (TP), Mean to Standard deviation Ratio (MSR), Contrast to Noise Ratio (CNR), and Equivalent Number of Looks (ENL) of the performance of our method was calculated for each 17 images. Then, the standard deviation (SD) and mean of EP, TP, MSR, CNR, and 
ENL of the performance of our method was compared to the famous denoising techniques (Table 2). When the structures of the image were more flattened and the edges inside the ROI were more blurred, these measurements had smaller values approximate to 0 . The TP and EP values in this table demonstrate the ability of our proposed method in preserving image structures and edges. Also, Figure 6 demonstrates the better comparison between different methods and our proposed method (in order to have a better illustration, the Mean ENL have been divided to 100 and the values of Mean TP and Mean EP have been multiplied to 10).

At first, the mentioned data consisting of 60 EDI-OCT images were manually segmented by a retinal ophthalmologist, then the automatic segmentation results of the choroid were compared to the manual segmentations applying Dice's Coefficient (DSC). DSC is a statistical metric for comparing the similarity between two samples presented by Thorvald Sørensen and Lee Raymond Dice (25), respectively in 1948 and 1945. Its formula (given two sets, $X$ and $Y$ ) is as the following:

See supplemental file for formula 3

The calculated mean DSC was $92.14 \% \pm 3.30 \%$ for our method. In order to compare our method with proposed method by Mazzaferri et al (17), we implemented their open-source algorithm on our dataset, the mean DSC of their proposed method was calculated as $55.75 \% \pm 14.54 \%$. Figure 7 shows the manual, proposed automatic and Mazzaferri's automatic segmentation results of the choroid.

\section{Discussion}

The techniques based on graph searching have been widely used for automatic segmentation of the choroid $(5,7,17,26-39)$. In this paper, a new method, based on curvelet transform and graph theory, was implemented for the automatic segmentation of the choroidal layer in OCT images from normal and abnormal OCT B-Scan images. The preprocessing and speckle noise removal of OCT images are important and directly affect the results of automatic segmentations. For this reason, a (2D) curveletbased K-SVD (15) algorithm was used to suppress the speckle noise and maintain the subtle features of OCT images. Also, due to the unwanted blurring effect of the K-SVD-based denoising method, the resulted images were deblurred by the Lucy-Richardson's deconvolution algorithm (13) and it provided improved output images when compared to the former studies (Table 2) by taking the EP, TP, MSR, CNR, and ENL measures into 17 SD-OCT retinal images. This deconvolution-based method utilized an estimated PSF along with transversal directions. As shown in Figure 4, the mean TP, mean EP, mean MSR and mean CNR were increased when compared to five other presented methods. Also, the value of the EP indicated that the proposed, method not only removed the speckle noise but also kept the edges very well. After preprocessing the OCT Images, as described in methods' session, the graph search based method was applied to automatic segmentation of the ICB and OCB. Figure 8 shows the results of our segmentation method with and without preprocessing step.

We have compared the manual choroidal segmentations of two expert raters with the results of proposed automatic segmentation algorithm on OCT images. The agreement between the automatic algorithm and 
the raters was significant and acceptable. An advantage of the presented method in comparison with other proposed techniques is that our method performed well in both high and low quality images but most of the other identical methods were efficient only in terms of the images with good quality. The raters stated that identification of the OCB in most of the scans was a less challenging task. It was observed that weak image intensity of $\mathrm{OCB}$ and inhomogeneous intensity of this layer caused a less disagreement between the manual and automatic segmentations on some of the OCT images. Since the used data set was not equal in different presented automatic segmentation methods, so there couldn't be a valid comparison between the presented method and the methods mentioned in the Table1. Therefore, the results of ten methods presented in Table 1 are generally discussed here. As, shown in this Table, the minimum presented DSC is $78 \% \pm 8 \%(39)$ and the maximum presented DSC is $97.35 \% \pm 2.3 \%$ (8). The average of DSC for these 10 methods was $90.56 \% \pm 3 \%$ while the mean DSC of our proposed method was calculated to be $92.14 \% \pm 3.30 \%$. Therefore, the proposed automatic segmentation method demonstrated the significant potential for getting a higher DSC rather than previous methods that mentioned in this paper. The comparison of the results of the automatic method with manual segmentations raised good agreement for both of the normal and abnormal cases.

\section{Conclusion}

The introduced method in this paper could provide easier and faster segmentation of the choroid in clinical trials that can improve the diagnosis of the choroid-related eye diseases. This algorithm is not so complicated and does not need any training, also, it gives precise results even in presence of high speckle noise. This method can be useful in studying the choroid layer in both healthy and diseased eyes in a large-scale and following up eye patients with choroidal disorders. Future development of this method will be done by extending the $2 \mathrm{D}$ method to $3 \mathrm{D}$ and improving the segmentation of this layer and also increase our data set.

\section{Declarations}

\section{Ethics approval and consent to participate}

This study was approved by the Regional Ethics Committee of Tabriz University of Medical Sciences (TUMS) (No: IR.TBZMED.REC.1397.008). Also, the written consent to participate form was used in this study and all the participants were adults (>18) who provided the written consent to participate.

\section{Consent for publication}

Not applicable.

\section{Availability of data and materials}


The datasets used in this study are available from the corresponding author upon reasonable request.

\section{Competing interests}

The authors declare that they have no known competing for financial interests or personal relationships that could have appeared to influence the work reported in this paper. The authors alone are responsible for the content and writing of the article.

\section{Funding}

Financial support for this study was provided by a grant from Research Deputy of Tabriz University of Medical Sciences with the grant number 59524. This research did not receive any specific grant from funding agencies in the public, commercial, or not for profit sectors.

\section{Authors' contributions}

AP and MA segmented the choroid manually in the used dataset. RAE and ME, designed the study, collected the data, worked on the proposed method, interpreted and analyzed the data, drafted and critically reviewed the manuscript. SHR, was responsible for the overall supervision of the work, as a corresponding author. All authors read and approved the final manuscript.

\section{Acknowledgments}

We thank all the patients who participated in this study. Also thanks from Hossein Najafzadeh for useful comments on code programming of the segmentation method.

\section{Abbreviations}

OCT: Optical Coherence Tomography

EDI-OCT: Enhanced Depth Imaging- Optical Coherence Tomography

ICB: Inner Choroidal Boundary

OCB: Outer Choroidal Boundary

DSC: Dice Similarity Coefficient

TP: Texture Preservation

ROI: Region of Interest 
PSF: Point Spread Function

AMD: Age-related Macular Degeneration

\section{References}

1.Nickla DL, Wallman J. The multifunctional choroid. Progress in retinal and eye research. 2010;29(2):144-68.

2.Wang J, Gao X, Huang W, Wang W, Chen S, Du S, et al. Swept-source optical coherence tomography imaging of macular retinal and choroidal structures in healthy eyes. BMC ophthalmology. 2015;15(1):122.

3.Huang W, Wang W, Zhou M, Chen S, Gao X, Fan Q, et al. Peripapillary choroidal thickness in healthy Chinese subjects. BMC ophthalmology. 2013;13(1):23.

4.Moussa M, Sabry D, Soliman W. Macular choroidal thickness in normal Egyptians measured by swept source optical coherence tomography. BMC ophthalmology. 2016;16(1):138.

5.Danesh H, Kafieh R, Rabbani H, Hajizadeh F. Segmentation of choroidal boundary in enhanced depth imaging OCTs using a multiresolution texture based modeling in graph cuts. Computational and mathematical methods in medicine. 2014;2014.

6.Behdad B, Rahmani S, Montahaei T, Soheilian R, Soheilian M. Enhanced depth imaging OCT (EDI-OCT) findings in acute phase of sympathetic ophthalmia. International ophthalmology. 2015;35(3):433-9.

7.Alonso-Caneiro D, Read SA, Collins MJ. Automatic segmentation of choroidal thickness in optical coherence tomography. Biomed Opt Express. 2013;4(12):2795-812.

8.Masood S, Fang R, Li P, Li H, Sheng B, Mathavan A, et al. Automatic Choroid Layer Segmentation from Optical Coherence Tomography Images Using Deep Learning. Scientific reports. 2019;9(1):3058.

9.Loupas T, McDicken W, Allan PL. An adaptive weighted median filter for speckle suppression in medical ultrasonic images. IEEE transactions on Circuits and Systems. 1989;36(1):129-35.

10.Portilla J, Strela V, Wainwright MJ, Simoncelli EP, editors. Adaptive Wiener denoising using a Gaussian scale mixture model in the wavelet domain. Image processing, 2001 proceedings 2001 international conference on; 2001: IEEE.

11.George A, Dilienseger J, Weber M, Pechereau A, editors. Optical coherence tomography image processing. Investigative Ophthalmology \& Visual Science; 2000: ASSOC RESEARCH VISION OPHTHALMOLOGY INC 9650 ROCKVILLE PIKE, BETHESDA, MD 20814-3998 USA. 
12. Herzog A, Boyer KL, Roberts C. Robust extraction of the optic nerve head in optical coherence tomography. Computer Vision and Mathematical Methods in Medical and Biomedical Image Analysis: Springer, 2004. p. 395-407.

13.Koozekanani D, Boyer $K$, Roberts $C$. Retinal thickness measurements from optical coherence tomography using a Markov boundary model. IEEE transactions on medical imaging. 2001;20(9):900-16.

14.0zcan A, Bilenca A, Desjardins AE, Bouma BE, Tearney GJ. Speckle reduction in optical coherence tomography images using digital filtering. JOSA A. 2007;24(7):1901-10.

15.Esmaeili M, Dehnavi AM, Rabbani H, Hajizadeh F. Speckle Noise Reduction in Optical Coherence Tomography Using Two-dimensional Curvelet-based Dictionary Learning. Journal of medical signals and sensors. 2017;7(2):86.

16. Hojjatoleslami S, Avanaki M, Podoleanu AG. Image quality improvement in optical coherence tomography using Lucy-Richardson deconvolution algorithm. Applied optics. 2013;52(23):5663-70.

17.Mazzaferri J, Beaton L, Hounye G, Sayah DN, Costantino S. Open-source algorithm for automatic choroid segmentation of OCT volume reconstructions. Scientific Reports. 2017;7:42112.

18.Lucy LB. An iterative technique for the rectification of observed distributions. The astronomical journal. 1974;79:745.

19.Richardson WH. Bayesian-based iterative method of image restoration. JOSA. 1972;62(1):55-9.

20.Laasmaa M, Vendelin M, Peterson P. Application of regularized Richardson-Lucy algorithm for deconvolution of confocal microscopy images. Journal of microscopy. 2011;243(2):124-40.

21.Starck J-L, Pantin E, Murtagh F. Deconvolution in astronomy: A review. Publications of the Astronomical Society of the Pacific. 2002;114(800):1051.

22.Chiu SJ, Li XT, Nicholas P, Toth CA, Izatt JA, Farsiu S. Automatic segmentation of seven retinal layers in SDOCT images congruent with expert manual segmentation. Optics express. 2010;18(18):19413-28.

23.Dijkstra EW. A note on two problems in connexion with graphs. Numerische mathematik. 1959;1(1):269-71.

24.Fang L, Li S, Nie Q, Izatt JA, Toth CA, Farsiu S. Sparsity based denoising of spectral domain optical coherence tomography images. Biomed Opt Express. 2012;3(5):927-42.

25.Dice LR. Measures of the amount of ecologic association between species. Ecology. 1945;26(3):297302.

26.Chen Q, Fan W, Niu S, Shi J, Shen H, Yuan S. Automated choroid segmentation based on gradual intensity distance in HD-OCT images. Optics express. 2015;23(7):8974-94. 
27.Chen Q, Niu S, Fang W, Shuai Y, Fan W, Yuan S, et al. Automated choroid segmentation of threedimensional SD-OCT images by incorporating EDI-OCT images. Computer Methods and Programs in Biomedicine. 2017.

28.Gerendas BS, Waldstein SM, Simader C, Deak G, Hajnajeeb B, Zhang L, et al. Three-dimensional automated choroidal volume assessment on standard spectral-domain optical coherence tomography and correlation with the level of diabetic macular edema. American journal of ophthalmology. 2014;158(5):1039-48. e1.

29.Hu Z, Wu X, Ouyang Y, Ouyang Y, Sadda SR. Semiautomated Segmentation of the Choroid in SpectralDomain Optical Coherence Tomography Volume Scans. Investigative Ophthalmology \& Visual Science. 2013;54(3):1722-9.

30.Hussain MA, Bhuiyan A, Ishikawa H, Smith RT, Schuman JS, Kotagiri R. An Automated Method for Choroidal Thickness Measurement from Enhanced Depth Imaging Optical Coherence Tomography Images. Computerized Medical Imaging and Graphics. 2018.

31.Kajić V, Esmaeelpour M, Považay B, Marshall D, Rosin PL, Drexler W. Automated choroidal segmentation of $1060 \mathrm{~nm}$ OCT in healthy and pathologic eyes using a statistical model. Biomed Opt Express. 2012;3(1):86-103.

32.Lee S, Fallah N, Forooghian F, Ko A, Pakzad-Vaezi K, Merkur AB, et al. Comparative Analysis of Repeatability of Manual and Automated Choroidal Thickness Measurements in Nonneovascular AgeRelated Macular DegenerationChoroidal Thickness Measurements in AMD. Investigative ophthalmology \& visual science. 2013;54(4):2864-71.

33.Lu H, Boonarpha N, Kwong MT, Zheng Y, editors. Automated segmentation of the choroid in retinal optical coherence tomography images. Engineering in Medicine and Biology Society (EMBC), 2013 35th Annual International Conference of the IEEE; 2013: IEEE.

34.Salafian B, Kafieh R, Rashno A, Pourazizi M, Sadri S. Automatic segmentation of choroid layer in edi oct images using graph theory in neutrosophic space. arXiv preprint arXiv:181201989. 2018.

35.Shi F, Tian B, Zhu W, Xiang D, Zhou L, Xu H, et al. Automated choroid segmentation in threedimensional< inline-formula $<$ mml: math display =. Journal of Biomedical Optics. 2016;21(12):126017.

36.Sui X, Zheng Y, Wei B, Bi H, Wu J, Pan X, et al. Choroid segmentation from optical coherence tomography with graph-edge weights learned from deep convolutional neural networks. Neurocomputing. 2017;237:332-41.

37.Tian J, Marziliano P, Baskaran M, Tun TA, Aung T, editors. Automatic measurements of choroidal thickness in EDI-OCT images. 2012 Annual International Conference of the IEEE Engineering in Medicine and Biology Society; 2012 Aug. 28 2012-Sept. 12012. 
38.Twa MD, Schulle KL, Chiu SJ, Farsiu S, Berntsen DA. Validation of Macular Choroidal Thickness Measurements from Automated SD-OCT Image Segmentation. Optometry \& Vision Science. 2016;93(11):1387-98.

39.Zhang L, Lee K, Niemeijer M, Mullins RF, Sonka M, Abramoff MD. Automated Segmentation of the Choroid from Clinical SD-OCTAutomated Segmentation of Choroid from SD-OCT. Investigative ophthalmology \& visual science. 2012;53(12):7510-9.

40.Kafieh R, Rabbani H, Foroohandeh M. Circular symmetric Laplacian mixture model in wavelet diffusion for dental image denoising. Journal of medical signals and sensors. 2012;2(2):103.

41.Luisier F, Blu T, Unser M. A new SURE approach to image denoising: Interscale orthonormal wavelet thresholding. IEEE Transactions on image processing. 2007;16(3):593-606.

42.Garvin MK, Abràmoff MD, Kardon R, Russell SR, Wu X, Sonka M. Intraretinal layer segmentation of macular optical coherence tomography images using optimal 3-D graph search. IEEE transactions on medical imaging. 2008;27(10):1495-505.

43.Bernardes R, Maduro C, Serranho P, Araújo A, Barbeiro S, Cunha-Vaz J. Improved adaptive complex diffusion despeckling filter. Optics express. 2010;18(23):24048-59.

44.Zhang L, Lee K, Niemeijer M, Mullins RF, Sonka M, Abramoff MD. Automated segmentation of the choroid from clinical SD-OCT. Investigative ophthalmology \& visual science. 2012;53(12):7510-9.

45.Vupparaboina KK, Nizampatnam S, Chhablani J, Richhariya A, Jana S. Automated estimation of choroidal thickness distribution and volume based on OCT images of posterior visual section.

Computerized Medical Imaging and Graphics. 2015;46:315-27.

46.Al-Bander B, Williams BM, Al-Taee MA, Al-Nuaimy W, Zheng Y, editors. A novel choroid segmentation method for retinal diagnosis using deep learning. 2017 10th International Conference on Developments in eSystems Engineering (DeSE); 2017: IEEE.

47.Chen M, Wang J, Oguz I, VanderBeek BL, Gee JC. Automated segmentation of the choroid in edi-oct images with retinal pathology using convolution neural networks. Fetal, Infant and Ophthalmic Medical Image Analysis: Springer, 2017. p. 177-84.

48.Wang C, Wang YX, Li Y. Automatic choroidal layer segmentation using markov random field and level set method. IEEE journal of biomedical and health informatics. 2017;21(6):1694-702.

\section{Tables}

Table. 1. A summary of automatic segmentation methods 


\begin{tabular}{|c|c|c|c|c|}
\hline & $\begin{array}{c}\text { Author \& } \\
\text { year }\end{array}$ & Dataset and samples & Segmentation method & Results \\
\hline 1 & $\begin{array}{c}\text { Tian et al. } \\
\text { (37) } \\
2012 \\
\end{array}$ & $\begin{array}{l}45 \text { B-scan from } 45 \text { healthy adult } \\
\text { subjects. }\end{array}$ & $\begin{array}{l}\text { Gradient-based graph search and } \\
\text { dynamic programming. }\end{array}$ & $\begin{array}{c}\text { Mean Dice } \\
\text { coefficient }= \\
90.5 \% \pm 3 \%\end{array}$ \\
\hline 2 & $\begin{array}{c}\text { Zhang et al. } \\
(44) \\
2012 \\
\end{array}$ & $\begin{array}{l}24 \text { normal subjects were imaged } \\
\text { twice on the same day. }\end{array}$ & Graph-based method. & $\begin{array}{c}\text { Dice } \\
\text { coefficient }= \\
78 \% \pm 8 \% \\
\end{array}$ \\
\hline 3 & $\begin{array}{l}\text { Lu et } \\
\text { al. } \\
2013^{(33)} \\
\end{array}$ & 30 adult subjects with diabetes. & $\begin{array}{l}\text { Gradient-based graph search, and } \\
\text { dynamic programming. }\end{array}$ & $\begin{array}{c}\text { Mean Dice } \\
\text { coefficient }= \\
92.7 \% \pm 3.6 \%\end{array}$ \\
\hline 4 & $\begin{array}{l}\text { Caneiro et al. } \\
\text { (7) } \\
2013\end{array}$ & $\begin{array}{l}90 \text { adult B-scans from } 15 \text { healthy } \\
\text { subjects and } 1083 \text { pediatric B- } \\
\text { scans from } 104 \text { healthy subjects. }\end{array}$ & $\begin{array}{l}\text { Dijkstra's shortest path algorithm, } \\
\text { directional weight, Edge filter, dual } \\
\text { brightness probability gradient. }\end{array}$ & $\begin{array}{c}\text { Mean Dice } \\
\text { coefficient }= \\
96.7 \% \pm 2.1 \%\end{array}$ \\
\hline 5 & $\begin{array}{l}\text { Vupparaboina } \\
\text { et al. (45) } \\
2015\end{array}$ & $\begin{array}{c}97 \text { B-scans per eye, from } 5 \text { healthy } \\
\text { adult subjects. }\end{array}$ & $\begin{array}{c}\text { Tensor voting, structural similarity } \\
\text { index, and eigenvalue analysis of the } \\
\text { Hessian matrix. }\end{array}$ & $\begin{array}{c}\text { Mean Dice } \\
\text { coefficient }= \\
95.47 \% \pm \\
1.73 \% \\
\end{array}$ \\
\hline 6 & $\begin{array}{l}\text { Shi et al. (35) } \\
2016\end{array}$ & 32 normal eyes. & $\begin{array}{l}\text { Three-dimensional graph search } \\
\text { method with the gradient-based cost. }\end{array}$ & $\begin{array}{c}\text { Mean Dice } \\
\text { coefficient }= \\
93.17 \% \pm \\
1.30 \% \\
\end{array}$ \\
\hline 7 & $\begin{array}{c}\text { Al-Bander et } \\
\text { al. (46) } \\
2017 \\
\end{array}$ & 169 EDI-OCT images & $\begin{array}{l}\text { Deep learning algorithm } \\
\text { (convolutional neural network) }\end{array}$ & $\begin{array}{c}\text { Dice } \\
\text { coefficient }= \\
89.76 \% \\
\end{array}$ \\
\hline 8 & $\begin{array}{c}\text { Chen et al. } \\
\text { (47) } \\
2017 \\
\end{array}$ & $\begin{array}{c}62 \text { EDI-OCT images of patients } \\
\text { with age-related macular } \\
\text { degeneration. }\end{array}$ & $\begin{array}{l}\text { Convolutional Neural Networks } \\
\text { (CNN). }\end{array}$ & $\begin{array}{c}\text { Mean Dice } \\
\text { coefficient = } \\
82 \% \pm 1 \% \\
\end{array}$ \\
\hline 9 & $\begin{array}{l}\text { Wang et al. } \\
\qquad \begin{array}{c}(48) \\
2017\end{array} \\
\end{array}$ & $\begin{array}{c}30 \text { images from } 30 \text { healthy } \\
\text { subjects with the ages between } 20 \\
\text { to } 85 \text { years. }\end{array}$ & $\begin{array}{l}\text { Markov random field and level set } \\
\text { methods. }\end{array}$ & $\begin{array}{c}\text { Mean Dice } \\
\text { coefficient }= \\
90 \% \pm 4 \% \\
\end{array}$ \\
\hline 10 & $\begin{array}{c}\text { Masood et al. } \\
\text { (8) } \\
2019\end{array}$ & $\begin{array}{l}525 \text { OCT images (25 scans from } \\
\text { every } 21 \text { subjects). }\end{array}$ & $\begin{array}{l}\text { Deep learning and a series of } \\
\text { morphological operations. }\end{array}$ & $\begin{array}{c}\text { Mean Dice } \\
\text { coefficient }= \\
97.35 \% \pm \\
2.3 \%\end{array}$ \\
\hline
\end{tabular}

Table. 2. Mean and standard deviation of the EP, TP, MSR, CNR, and ENL for 17 SD-OCT retinal images by the use of 3D CWDL (40), Tikonov (41), MSBTD (42), K-SVD (43), K-SVD based DCUT (15), and proposed method. 


\begin{tabular}{|c|c|c|c|c|c|c|c|}
\hline & Original & 3D CWDL (40) & $\begin{array}{c}\text { Tikonov } \\
(41)\end{array}$ & MSBTD (42) & K-SVD (43) & $\begin{array}{l}\text { K-SVD based } \\
\text { DCUT (15) }\end{array}$ & $\begin{array}{l}\text { Proposed } \\
\text { Method }\end{array}$ \\
\hline $\begin{array}{c}\text { Mean } \pm \text { STD } \\
\text { (EP) }\end{array}$ & $1 \pm 0$ & $0.91 \pm 0.22$ & $0.71 \pm 0.45$ & $0.68 \pm 0.59$ & $0.63 \pm 0.33$ & $0.96 \pm 0.02$ & $\begin{array}{c}0.95 \pm \\
0.01 \\
\end{array}$ \\
\hline $\begin{array}{c}\text { Mean } \pm \text { STD } \\
\text { (TP) }\end{array}$ & $1 \pm 0$ & $0.41 \pm 0.52$ & $0.18 \pm 0.85$ & $0.12 \pm 0.87$ & $0.32 \pm 0.25$ & $0.75 \pm 0.05$ & $\begin{array}{c}0.96 \pm \\
0.02 \\
\end{array}$ \\
\hline $\begin{array}{c}\text { Mean } \pm \text { STD } \\
(\text { MSR) }\end{array}$ & $3.20 \pm 0.46$ & $14.45 \pm 4.85$ & $7.64 \pm 0.63$ & $14.76 \pm 4.75$ & $11.22 \pm 2.77$ & $14.36 \pm 3.85$ & $\begin{array}{c}29.33 \pm \\
20.18\end{array}$ \\
\hline $\begin{array}{c}\text { Mean } \pm \text { STD } \\
(\mathrm{CNR})\end{array}$ & $1.27 \pm 0.43$ & $7.31 \pm 3.63$ & $3.26 \pm 0.22$ & $4.76 \pm 1.54$ & $4.11 \pm 1.23$ & $7.81 \pm 3.54$ & $\begin{array}{c}31.07 \pm \\
19.78 \\
\end{array}$ \\
\hline $\begin{array}{c}\text { Mean } \pm \text { STD } \\
(\text { ENL) }\end{array}$ & $38.09 \pm 4.35$ & $2323.65 \pm 43.54$ & $74.34 \pm 4.32$ & $783.68+67.65$ & $132.01 \pm 7.32$ & $1983.08 \pm 39.42$ & $\begin{array}{c}69.51 \pm \\
138.12\end{array}$ \\
\hline
\end{tabular}

\section{Figures}

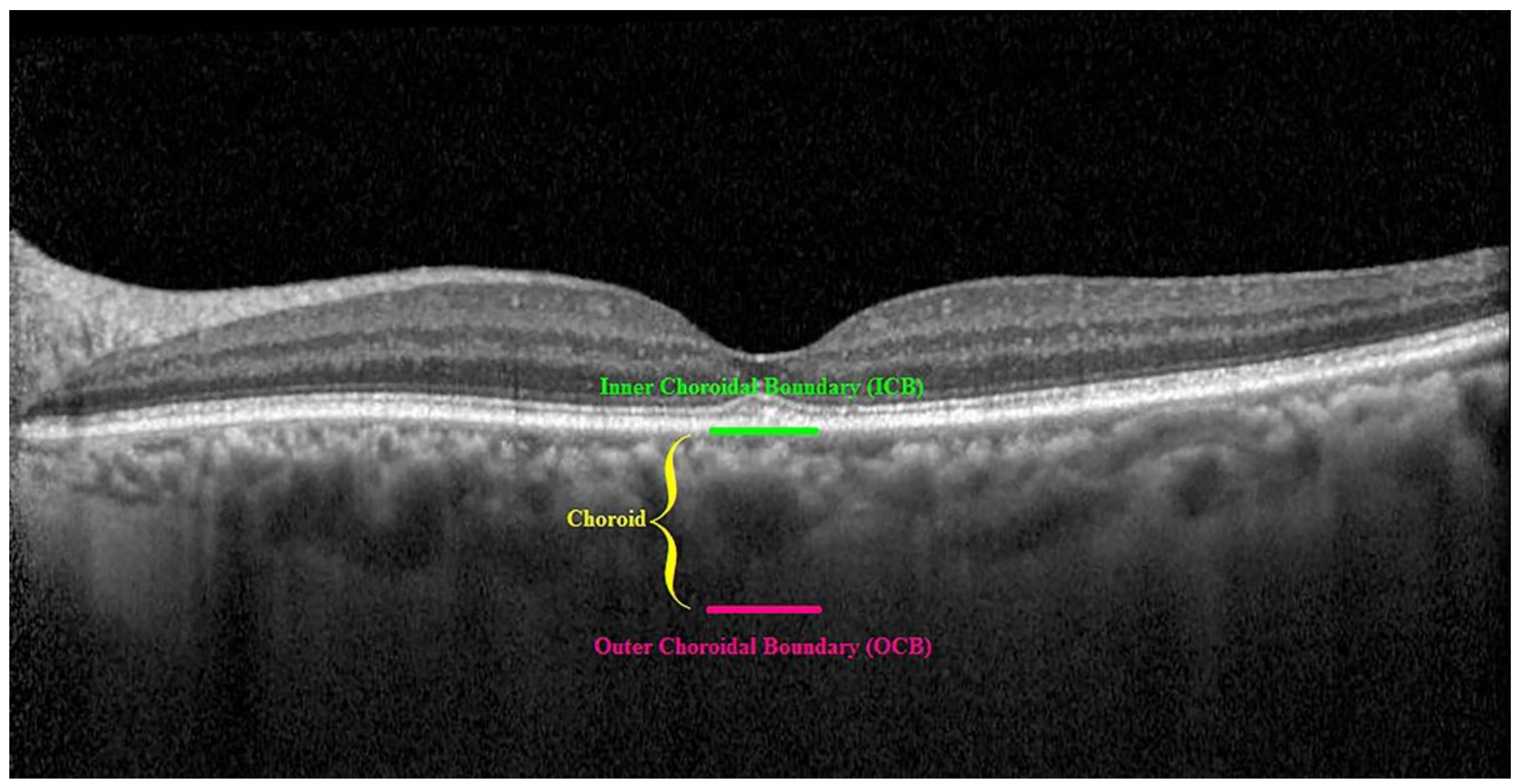

Figure 1

A sample of an EDI-OCT image of the choroid. 


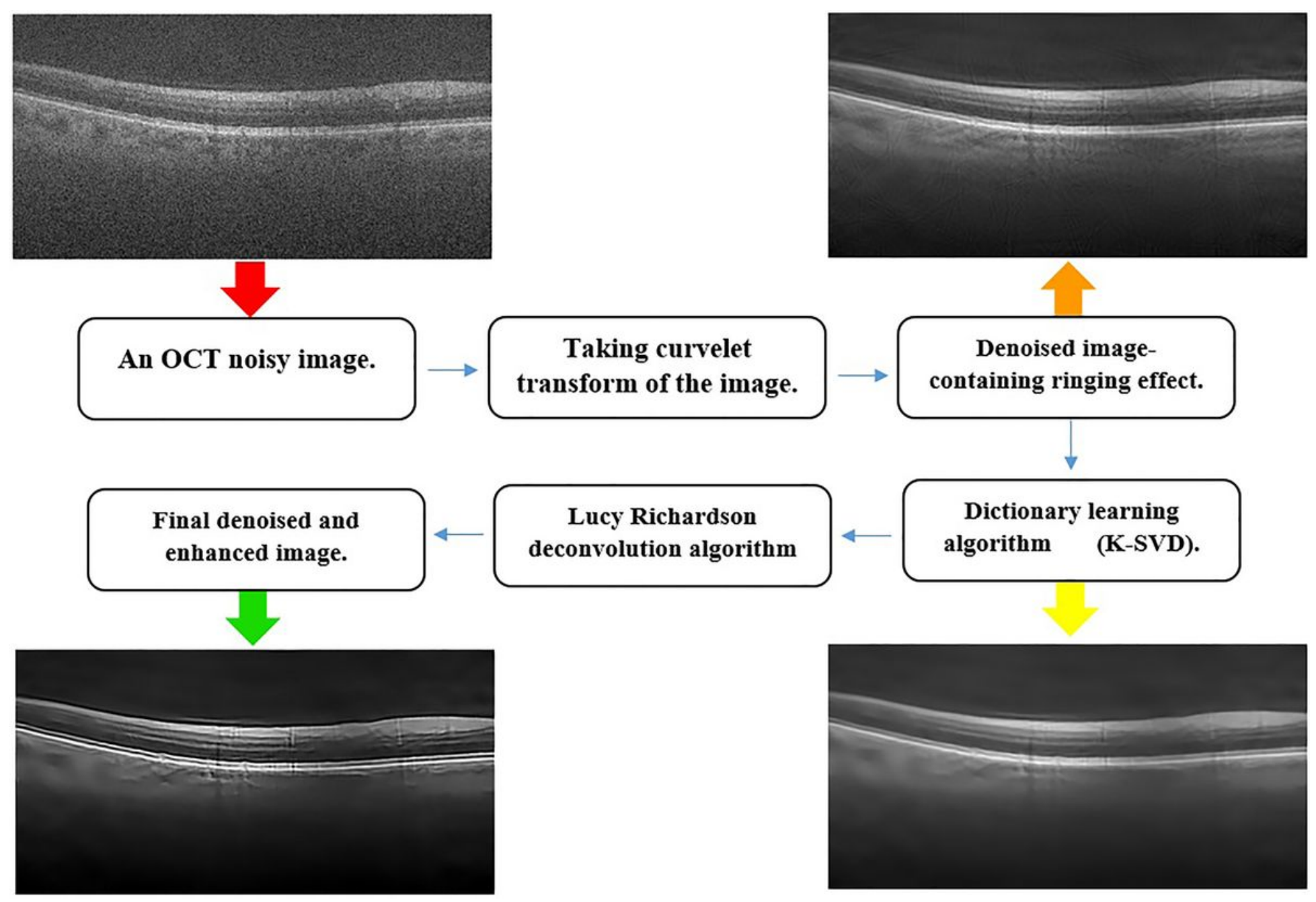

Figure 2

Block diagram of the preprocessing and image enhancement method. 
Figure 3

An example of automatic segmentation of ICB which is shown with the green line.
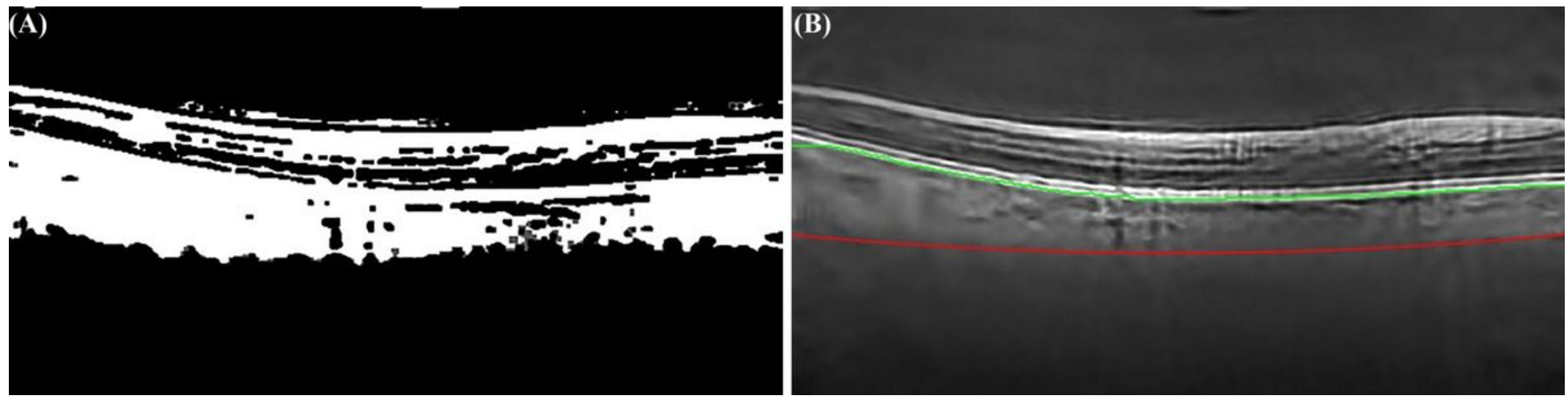

Figure 4

(A) A thresholded OCT image, (B) An example of automatic segmentation of OCB which is shown with the red line. 

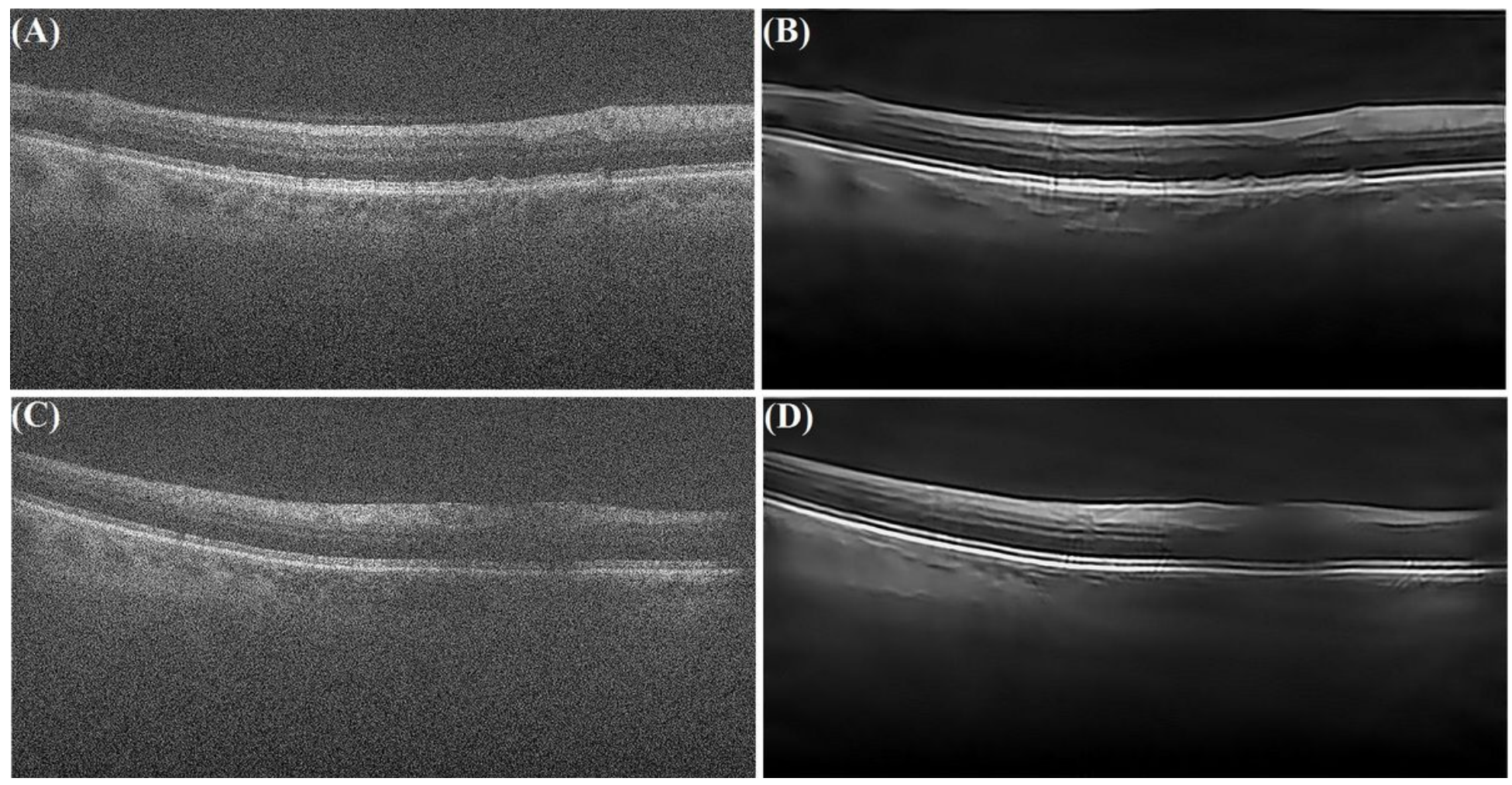

Figure 5

(A) OCT image1 containing speckle noise, (B) Enhanced OCT image1, (C) OCT image2 containing speckle noise, (D) Enhanced OCT image2.

25

20

15

10

5

0

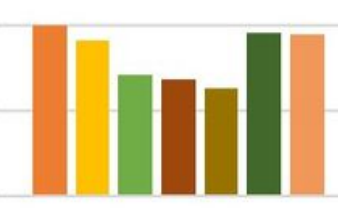

Mean EP

- Original

K-SVD [33]

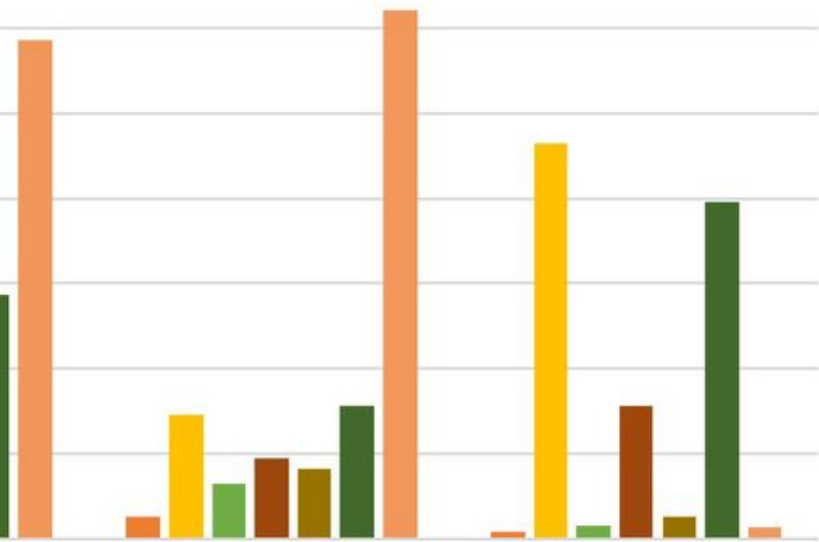

Mean CNR

Mean ENL
Mean MSR

Mean TP

- 3D CWDL [22]

- K-SVD based DCUT [2] a Proposed Method

Figure 6

Shows the mean of the EP, TP, MSR, CNR, and ENL for 17 SD-OCT retinal images by the use of 3D CWDL (40), Tikonov (41), MSBTD (42), K-SVD (43), K-SVD based DCUT (15), and proposed method. 

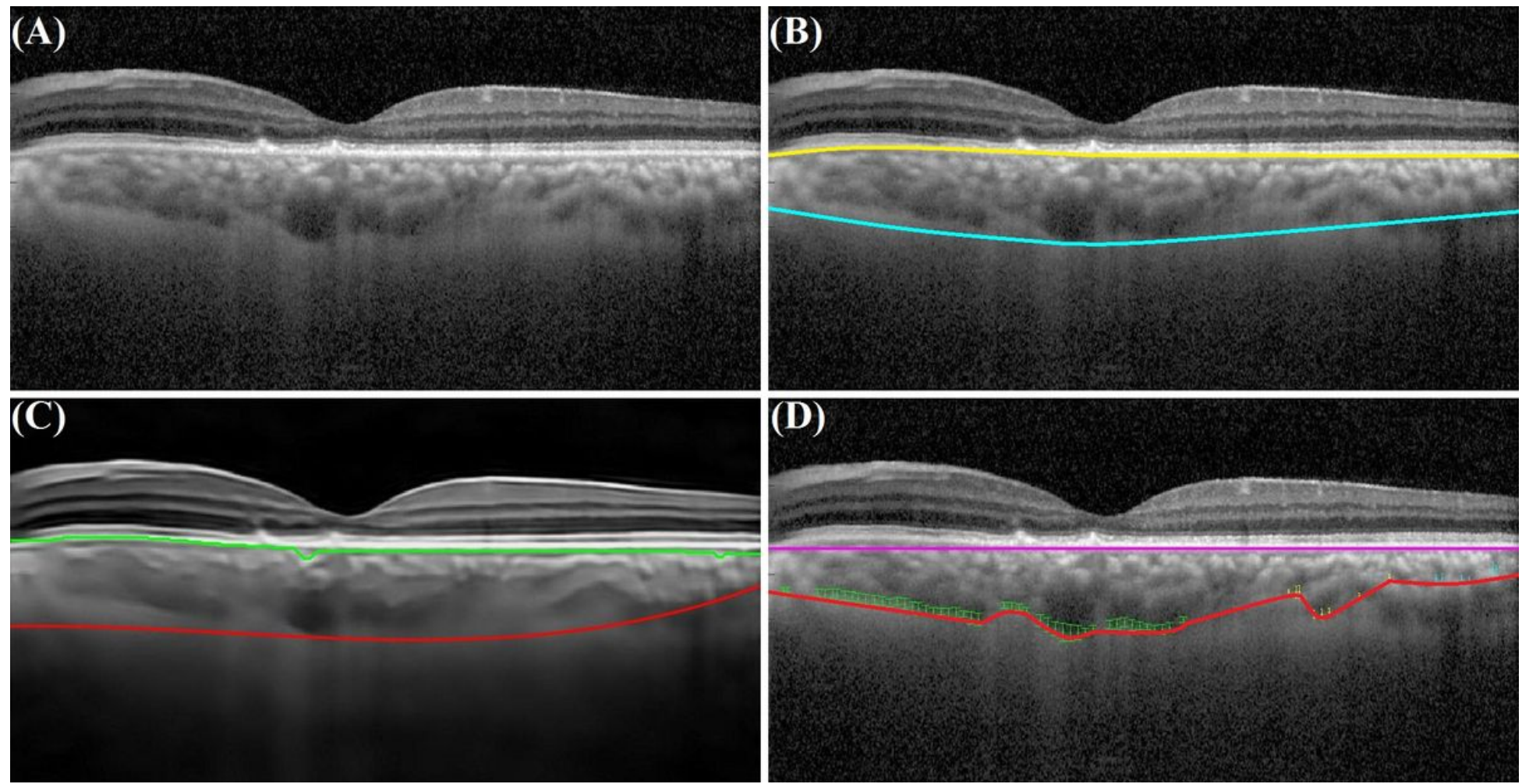

Figure 7

(A) Noisy EDI-OCT image, (B) Manual segmentation of choroidal layer by an expert, (C) Automatic segmentation of choroid by our proposed method, (D) Automatic segmentation of choroid by the use of proposed algorithm by Mazzaferri et al (17).

100

90

80

70

60

50

40

30

20

10

0

\section{$71.99 \%$}
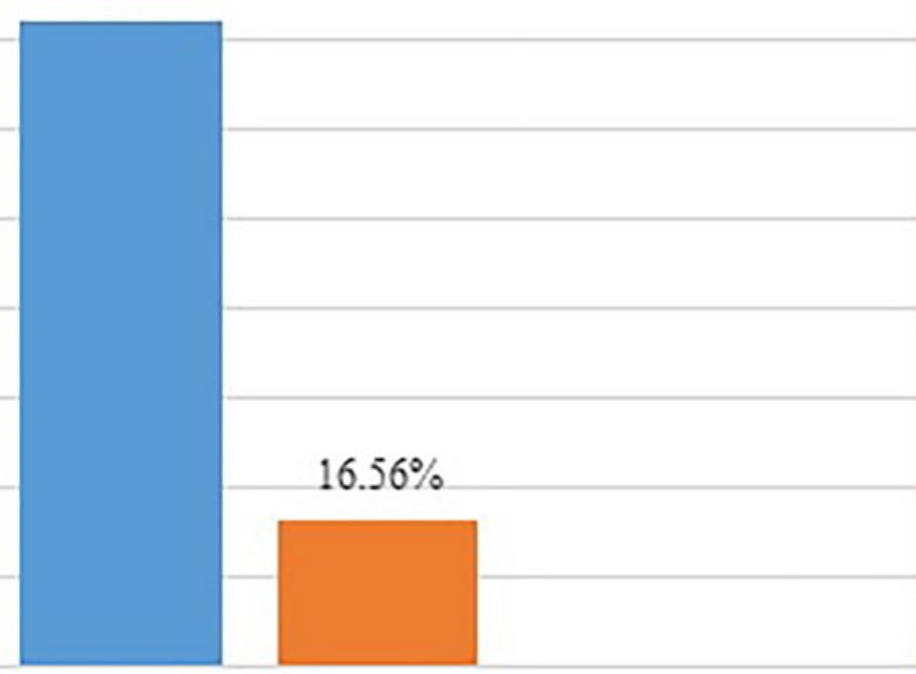

\section{$92.14 \%$}

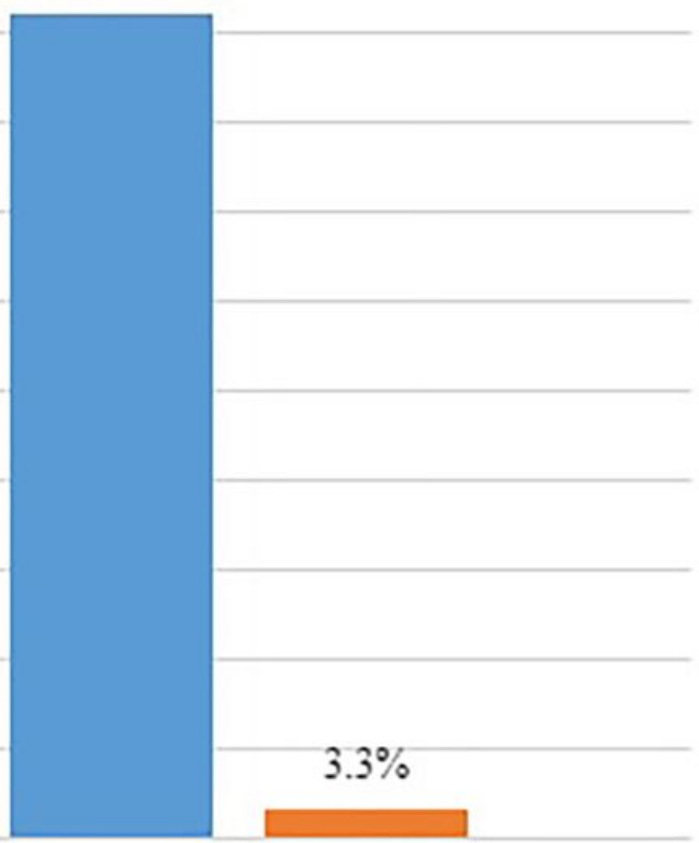

Results of segmentations with out preprocessing step. Results of segmentations after preprocessing step.

$\square$ Mean DSC $\quad$ Standard Deviation 
Figure 8

The results of our proposed automatic segmentation method with and without preprocessing step.

\section{Supplementary Files}

This is a list of supplementary files associated with this preprint. Click to download.

- formula3.JPG

- formula2.JPG

- formula1.JPG 